\title{
The DIRC Detectors at the PANDA Experiment*
}

A. Lehmann ${ }^{\dagger 3}$, R. Dzhygadlo ${ }^{1}$, A. Gerhardt ${ }^{1}$, K. Götzen ${ }^{1}$, G. Kalicy ${ }^{1}$, M. Krebs ${ }^{1}$, H. Kumawat ${ }^{1}$, D. Lehmann ${ }^{1}$, M. Patsyuk ${ }^{1}$, K. Peters ${ }^{1}$, G. Schepers ${ }^{1}$, L. Schmitt ${ }^{1}$, C. Schwarz ${ }^{1}$, J. Schwiening ${ }^{1}$, M. Traxler ${ }^{1}$, M. Zühlsdorf ${ }^{1}$, V. Kh. Dodokhov ${ }^{2}$, A. Britting ${ }^{3}$, W. Eyrich ${ }^{3}$, F. Uhlig ${ }^{3}$, M. Düren ${ }^{4}$, E. Etzelmüller ${ }^{4}$, K. Föhl $^{4}$, A. Hayrapetyan ${ }^{4}$, B. Kröck ${ }^{4}$, O. Merle ${ }^{4}$, J. Rieke ${ }^{4}$, E. Cowie ${ }^{5}$, T. Keri ${ }^{5}$,

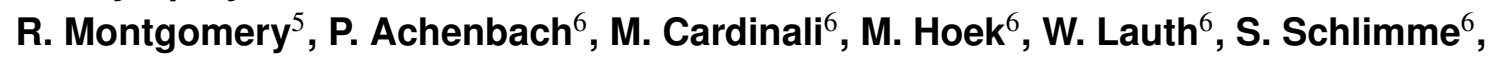
C. Sfienti ${ }^{6}$, M. Thiel ${ }^{6}$, P. Bühler ${ }^{7}$, L. Gruber ${ }^{7}$, J. Marton ${ }^{7}$, K. Suzuki ${ }^{7}$

${ }^{1}$ GSI Helmholtzzentrum für Schwerionenforschung GmbH, Darmstadt, Germany

${ }^{2}$ Joint Institute for Nuclear Research, Dubna, Russia

${ }^{3}$ Friedrich Alexander-University of Erlangen-Nuremberg, Erlangen, Germany

${ }^{4}$ II. Physikalisches Institut, Justus Liebig-University of Giessen, Giessen, Germany

${ }^{5}$ University of Glasgow, Glasgow, United Kingdom

${ }^{6}$ Institut für Kernphysik, Johannes Gutenberg-University of Mainz, Mainz, Germany

${ }^{7}$ Stefan Meyer Institut für subatomare Physik, Austrian Academy of Sciences, Vienna, Austria

E-mail: albert.lehmannephysik.uni-erlangen.de

PANDA is an experiment at the new FAIR facility at GSI and will, among other physics goals, perform charmonium spectroscopy and search for gluonic excitations using high luminosity antiproton beams up to $15 \mathrm{GeV} / \mathrm{c}$. A high performance particle identification system applying DIRC detectors will allow pion/kaon separation up to $4 \mathrm{GeV} / \mathrm{c}$. A Barrel DIRC with fused silica radiator bars or plates will surround the target at a radial distance of $48 \mathrm{~cm}$ and will cover a polar angle range of 22 to 140 degrees; a novel Endcap Disk DIRC built of a segmented fused silica disk of $210 \mathrm{~cm}$ diameter will be installed in the forward region to cover the polar angles from 5 to 22 degrees. The design of the optics and the readout of both DIRCs will be presented in this paper. Different prototypes were tested in particle beams. The performance of the latest prototypes, which are close to the final DIRC design, are discussed and compared to the PANDA requirements.

Technology and Instrumentation in Particle Physics 2014

2-6 June, 2014

Amsterdam, the Netherlands

${ }^{*}$ This work is supported by BMBF and GSI.

† Speaker. 


\section{Introduction}

PANDA [U] is one of the pillar experiments at the new FAIR (Facility for Antiproton and Ion Research) accelerator at GSI in Darmstadt, Germany. In a dedicated High Energy Storage Ring (HESR) up to $10^{11}$ antiprotons can be stored and accelerated to a momentum of 1.5 to 15 $\mathrm{GeV} / \mathrm{c}$. An excellent momentum resolution of up to $10^{-5}$ and a luminosity of up to $2 \cdot 10^{32} \mathrm{~cm}^{-2} \mathrm{~s}^{-1}$ will allow hadron physics measurements with unprecedented accuracy. Among other goals the physics program of PANDA will be charmonium spectroscopy and the search for exotics states like glueballs and hybrids. For most of these goals an excellent particle identification is required, in particular the separation of pions and kaons up to a momentum of $4 \mathrm{GeV} / \mathrm{c}$.

The backbone of the hadron identification system of PANDA will consist of two Cherenkov detectors: a barrel DIRC and a novel endcap disk DIRC (see Figure W). In DIRC detectors a relativistic charged particle traversing a high refractive index radiator produces Cherenkov photons which are usually guided to a focal plane outside of the main detector by internal total reflection along the radiator [[]]. This is somewhat different in PANDA, where for spatial reasons the focal planes of the DIRCs will be placed inside the magnetic field of the solenoid of up to 2 Tesla. This requires photon sensors capable of dealing with this field.

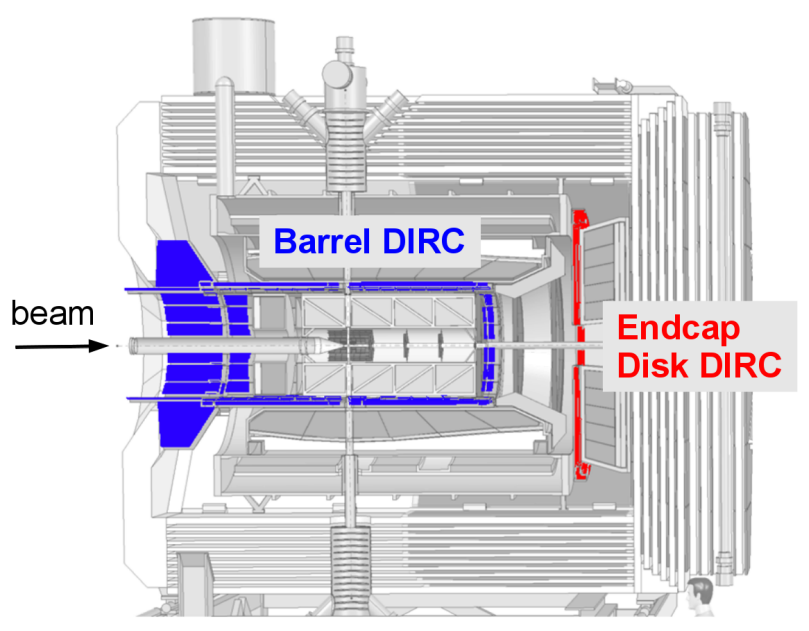

Figure 1: View of the PANDA Target Spectrometer. The positions of the Barrel DIRC and the Endcap Disk DIRC are indicated in blue and red, respectively.

\section{Special Challenge Photon Sensors}

The preferred sensors for the PANDA DIRC detectors are microchannel-plate (MCP) PMTs, because they allow single photon detection in high magnetic fields with an excellent time resolution of $<50 \mathrm{ps}$ [3]. However, until recently these devices had serious aging problems caused by feedback ions hitting and damaging the photo cathode. This results in a diminishing quantum efficiency (QE) as the integrated anode charge increases. Due to recent developments in the MCP design a huge improvement in the lifetime was accomplished [团]. The latest MCP-PMTs of PHOTONIS are suitable of accumulating anode charges of $>6 \mathrm{C} / \mathrm{cm}^{2}$ without a QE degradation (see Figure $\mathrm{Z}$ ). This would be sufficient for both PANDA DIRCs. 


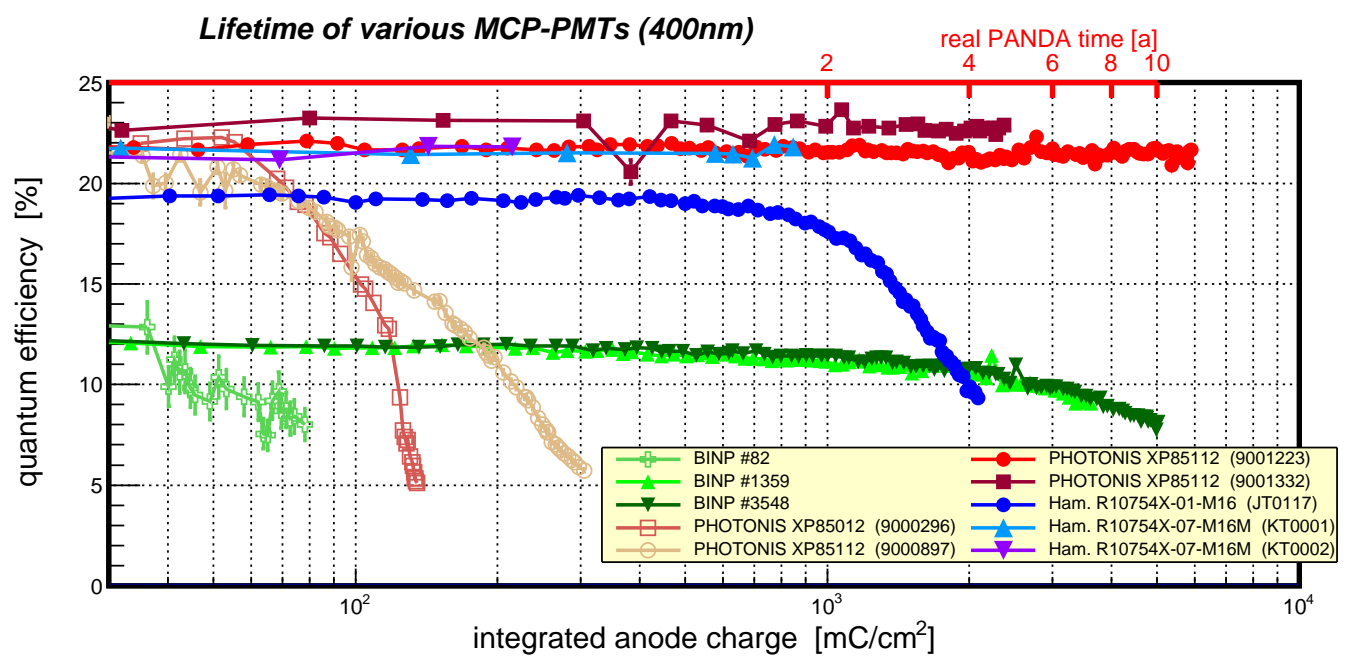

Figure 2: Comparison of the lifetime of different MCP-PMTs: QE as a function of the integrated anode charge at $400 \mathrm{~nm}$ [䧃]. The older MCP-PMTs are those with the open symbols, the new lifetime-enhanced devices are those with the solid symbols.

\section{Endcap Disk DIRC}

PANDA is a fixed-target experiment and many particles will be boosted to the forward hemisphere. To identify the particle types in the polar angle range from 5 to 22 degrees a novel DIRC design was developed using a synthetic fused silica radiator disk with $210 \mathrm{~cm}$ diameter and $20 \mathrm{~mm}$ thickness []]. The goal is a $3 \sigma \pi / K$ separation up to $4 \mathrm{GeV} / \mathrm{c}$. The Endcap Disk DIRC (EDD) has the shape of a regular dodecagon and will consist of four identical but optically seperated subdetectors (see Figure B]). At the inside of the disk a diamond-shaped hole is left open for the passage of the antiproton beam.

At each straight section of the outer rim of each disk quarter nine readout modules (ROMs) will be connected. A ROM consists of three $16 \mathrm{~mm}$ wide optical modules - a quartz bar and a focusing element (FEL) with an aluminized cylindrical surface - to focus the Cherenkov photons onto the readout plane which is equipped with a 2 inch MCP-PMT with a highly segmented anode of 3x100 pixels. Between the FEL and the MCP-PMT an optical wavelength filter will be installed to adjust the wavelength of detected photons to a band which reduces dispersion effects as well as enhances the sensor lifetime. E.g., between 385 and $460 \mathrm{~nm}$ an average number of 16 photons/track will be detected, which is sufficient to reconstruct the Cherenkov angle accurately enough for the $3 \sigma \pi / K$ separation aimed at (see also Fig. ( $)$ ). More details about the design of the EDD can be found in [目].

With the wavelength filter the anticipated average detected photon rate at each MCP anode is about $20 \mathrm{kHz}$. Assuming 10 years of PANDA running with 50\% duty cycle this will accumulate to an integrated anode charge of 5-6 $\mathrm{C} / \mathrm{cm}^{2}$. It is planned to digitize the anode signals of the MCP-PMTs with the TOFPET ASIC chip which probably has to be modified to process the short MCP-PMT signals. Since ASICs can be built in very compact sizes it is possible to directly connect it to the back of the MCP-PMT avoiding analog signal cables. The digitized information will then be sent to the PANDA data acquisition system with optical fiber cables. 

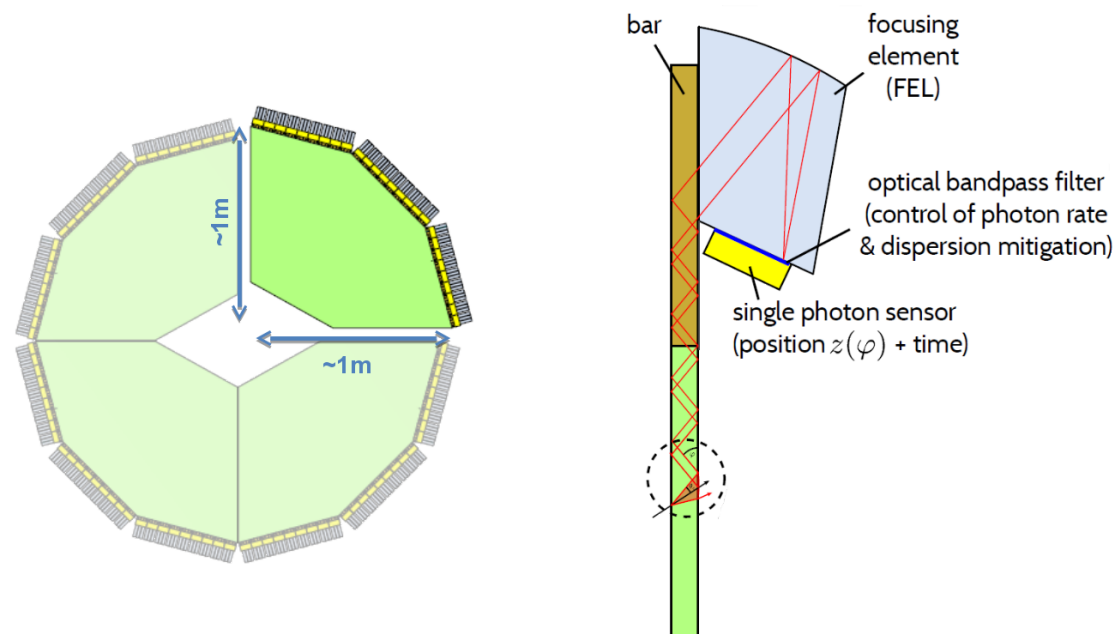

Figure 3: Schematic view of the EDD design: radiator disk subdivided in 4 quarters (left) and focusing optics with sensor (right).
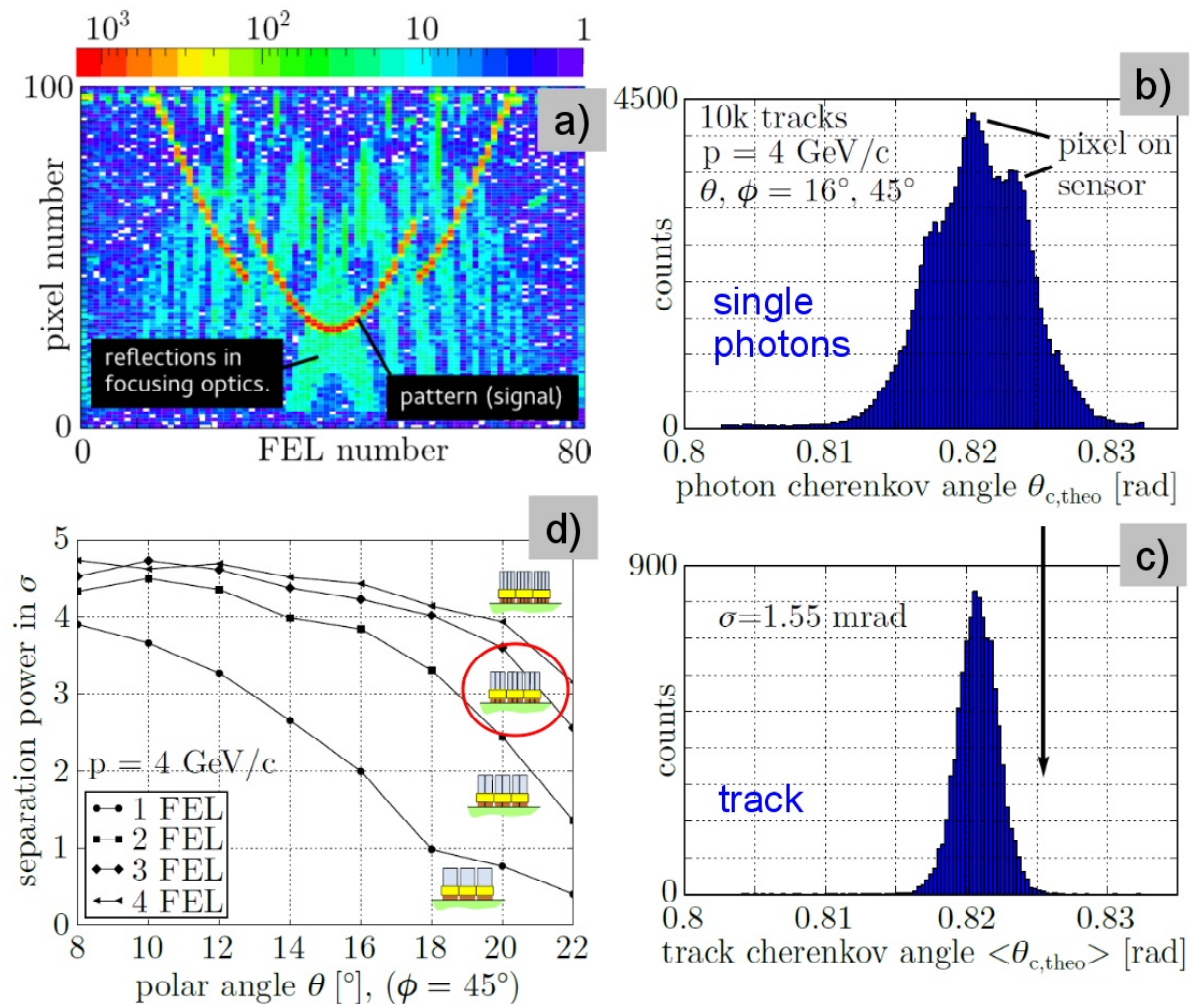

Figure 4: Simulated hit pattern and reconstruction results for the EDD [B]. The explanation of the individual plots is given in the text. 
The final design of the EDD was heavily guided by Monte Carlo simulations based on the Geant 4 framework. Surface losses at the optical connections, bulk attenuation, detector inefficiencies and dead times in the front end electronics were taken into account as well as the resolutions of the measured observables. Figure 1 shows an accumulated hit pattern of 10k simulated pions at a momentum of $4 \mathrm{GeV} / \mathrm{c}$ at a polar angle of 16 degree and an azimuthal angle of 45 degree. The background underneath the real signal pattern (a) stems from reflections in the focusing optics. From these patterns a theoretical single photon Cherenkov angle resolution of about $8 \mathrm{mrad}$ (b) was determined which translates to an average Cherenkov angle resolution per track of $<2 \mathrm{mrad}$ (c) assuming the above mentioned 16 detected photons/track. This kind of simulation was done for many polar angles and different designs of the optical modules [G]. The best compromise in separation power was obtained by using three FELs per MCP-PMT (d). A MCP pixel size of 0.5 $\mathrm{mm}$ is needed to exploit the full FEL resolution which defines the necessity of a highly segmented anode of the MCP-PMTs.

For a first evaluation of the concept a quarter segment of the EDD was built with $80 \%$ of its final size and a Borofloat radiator [ $[\mathbf{D}]$. Thirty plexiglass (PMMA) focusing elements equipped with thirty 16-channel linear array multianode PMTs (H10515B-100) were read out by HADES TRBs (Trigger and Readout Boards) [8]. This prototype was tested at the CERN PS T9 beam line with a mixed lepton/hadron beam of $3.5 \mathrm{GeV} / \mathrm{c}$. An external particle identification was provided by TOF and threshold Cherenkov counters.

The results of this test are shown in Figure [ 5 . In the cumulative hit patterns (data upper and lower left, simulations upper right) a clear distinction between protons and pions is observed which agrees well with the simulations. The single event hit patterns were analyzed by using shapes of the cumulative hit patterns as the base for a log-likelihood approach. From the plots (lower right) a clear distinction between pions and protons is visible. The amount of misidentified protons is less than $2 \%$ if more than 10 photons were detected. For the separation of pions and kaons much better optics and a better granularity of the PMT anodes will be needed. This test is planned with a full-size prototype of an EDD quarter which will be equipped with the final optics and electronics.
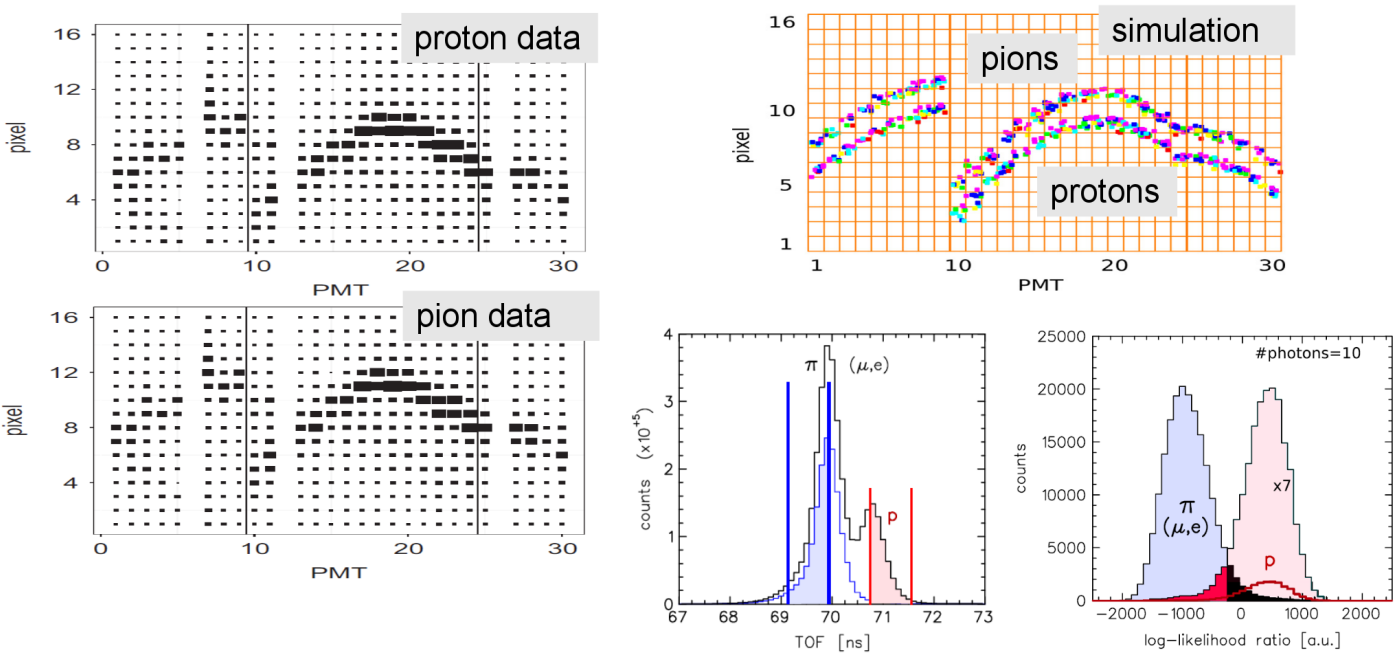

Figure 5: PID with an R\&D EDD prototype tested in a mixed hadron beam [प]. 


\section{Barrel DIRC}

The hadron identification in the polar angle range from 22 to 140 degrees will be done with a Barrel DIRC (BD) [9] which consists of 16 sectors of $17 \mathrm{~mm}$ thick fused silica radiators surrounding the interaction point at $48 \mathrm{~cm}$ radius (see Figure G). Currently two design options are being investigated:

1. Each sector is equipped with five $32 \mathrm{~mm}$ wide and $2400 \mathrm{~mm}$ long bars placed side-by-side and separated by a small air gap. At the downstream end a mirror is attached to each bar to reflect forward going Cherenkov photons towards the upstream readout end of the bars. This design is very similar to that of the BaBar DIRC [ए]]], except that there will be only a $30 \mathrm{~cm}$ deep expansion volume (EV) at the readout side. The EV will be either a tank filled with mineral oil or a fused-silica prism for each sector. To focus the photons onto the focal plane lenses attached to the bar end will be needed.

2. Each sector contains one wide plate with $160 \mathrm{~mm}$ width and $2400 \mathrm{~mm}$ length. The EV will be a $16 \mathrm{~cm}$ wide and $30 \mathrm{~cm}$ deep prism as above possibly equipped with high reflective index lenses at the entrance. Although it will be more complicated to reconstruct the Cherenkov angle this option is considered because of significantly lower fabrication costs since fewer surfaces have to be polished.

The focal plane will be equipped with an array of MCP-PMTs with roughly 10000-15000 readout channels to measure the position and arrival time of the Cherenkov photons. The MCP signals will be digitized with fast frontend boards, either PADIWA or NINO [प]], whose TDCs deliver a time stamp with $<20$ ps resolution (RMS) and a rough pulse height information by applying a time-over-threshold technique. The performance goals of these design scenarios are a single photon Cherenkov angle resolution of $8-9 \mathrm{mrad},>20$ detected photons for $\beta \approx 1$ particles, and a time resolution of $<100 \mathrm{ps}$. The ultimate goal of the $\mathrm{BD}$ is a $3 \sigma \pi / K$ separation up to $3.5 \mathrm{GeV} / \mathrm{c}$.

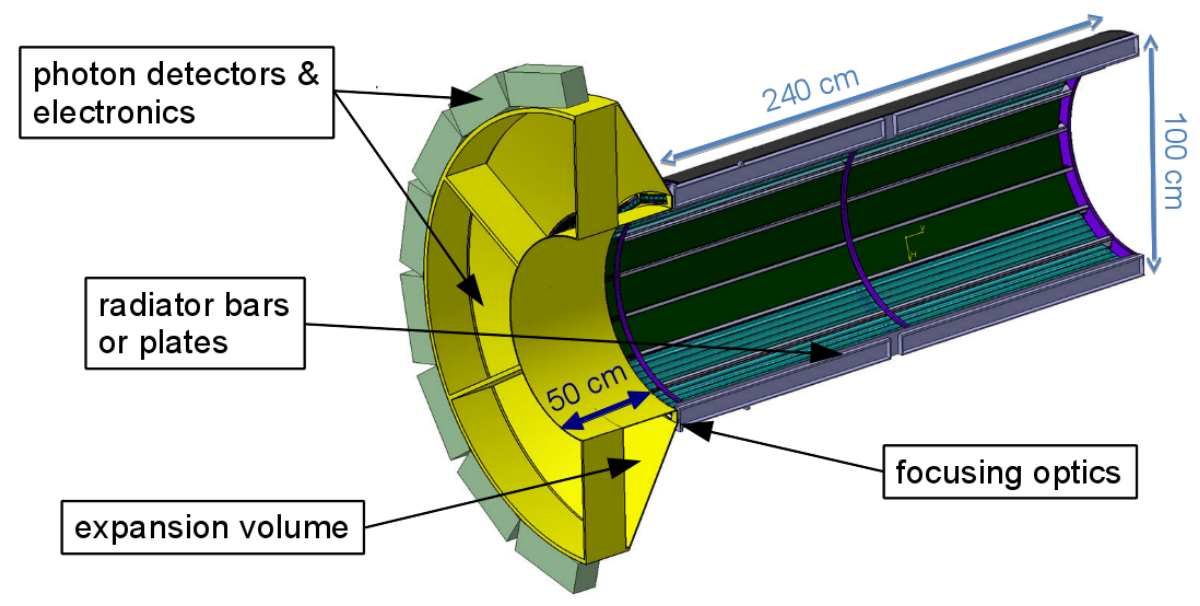

Figure 6: Schematic view of the PANDA Barrel DIRC base design.

In the BaBar DIRC the reconstruction of the Cherenkov angle was done with look-up tables. This approach can be applied in a similar way for the option with the narrow bar radiators [10]]. 
In the case of a wide radiator plate the reconstruction will be more complicated. The pattern recognition will be based on a log-likelihood analysis similar to the Belle II time imaging approach for their Time-Of-Propagation counter [123, 녀]. With this method simulations suggest a clean $\pi / K$ separation at $3.5 \mathrm{GeV} / \mathrm{c}$ even without focusing the photons (see Figure $\square$ ).

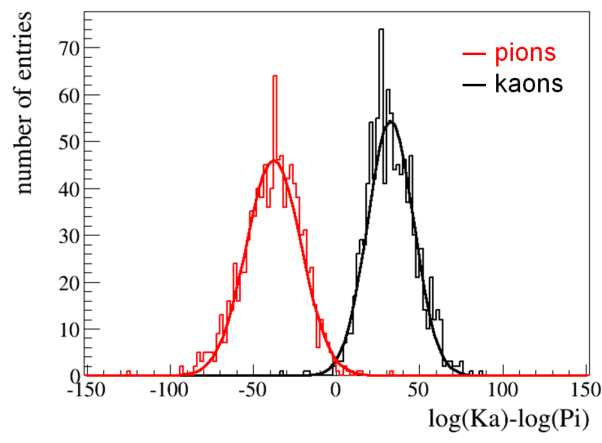

Figure 7: Log-likelihood difference for kaon and pion hypotheses for a sample of $3.5 \mathrm{GeV} / \mathrm{c}$ pions and kaons at a polar angle of $22^{\circ}$ [प]5].

The performance of several BD prototypes was investigated in experiments with particle beams at GSI and CERN. In these performance tests different configurations with bar/plate, with/without lens and with/without prism were studied. The setup of one of the latest tests [ए]] at the CERN PS T9 beamline with a radiator bar and a prism is shown in Figure 8 . The beam of up to $10 \mathrm{GeV} / \mathrm{c}$ was a mixture of leptons and hadrons; pions, kaons and protons were identified with TOF counters. The bars/lens/prism DIRC prototype was mounted on an adjustable table which could be rotated and changed in height. With this setup a wide range of beam-bar angles and positions with radiators from different vendors and lenses of various shapes were tested. The readout was done with a $3 \times 3$ array of 2 inch PHOTONIS XP85012 MCP-PMTs. Together with the trigger and the tracking detectors the signals of about 640 channels were digitized with HADES TRB boards [ [ $]$ ].

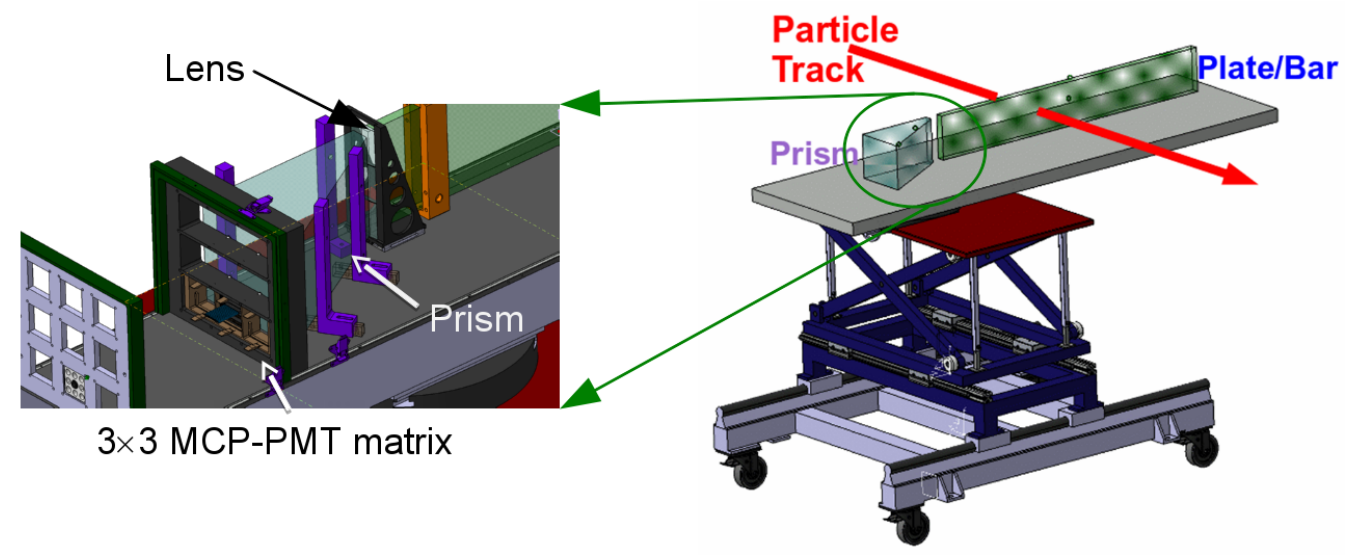

Figure 8: Schematic view of the prototype configuration at CERN in 2012.

Results of the latest prototype test at the CERN PS T9 beamline are shown in Figure 8 [प5]. The data were taken with a combination bar/lens/prism. The hit patterns are complicated due to reflections in the prism where the ring image is folded. However, as visible in the occupancy plots 
(a, b) the agreement between simulations and data is good. A single photon Cherenkov angle resolution of $13 \mathrm{mrad}$ was obtained (c) and an average number of detected photons/track of $>20$ was measured. This is very close to the design values for the BD given above.
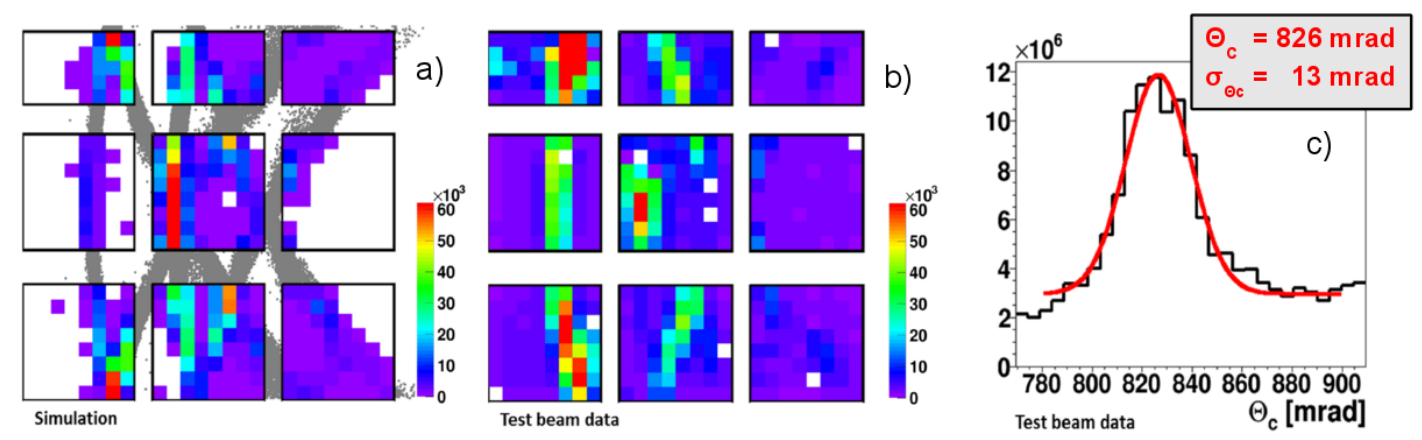

Figure 9: Occupancy plots of detected photons per MCP-PMT pixel for a combination bar/lens/prism at a beam-bar angle of $56^{\circ}$. The grey dots indicate the simulated Cherenkov pattern at the imaging plane while the colour boxes show the actual MCP-PMT responses for simulations (a) and data (b). In panel (c) the single photon Cherenkov angle resolution is shown.

\section{Summary}

The $\mathrm{R} \& \mathrm{D}$ work for the PANDA DIRC detectors is well on track. The design phase is almost completed and prototype tests are ongoing. Simulations indicate that with the final Endcap Disk DIRC design using three FELs per MCP-PMT a Cherenkov angle resolution per track of $<2 \mathrm{mrad}$ will be obtained which is sufficient for a $3 \sigma \pi / K$ separation up to $4 \mathrm{GeV} / \mathrm{c}$. Beam tests with simplified prototypes show very promising results although an ultimate test with a full-size and fully equipped EDD quarter still has to be performed. For this the delivery of MCP-PMTs with highly segmented anodes and their readout are still pending. With the application of a bar/lens/prism combination the construction of a compact and fast focusing Barrel DIRC appears feasible. Prototype tests show that the performance of the radiator bar design for the BD is close to the PANDA requirements. A single photon Cherenkov angle resolution of $13 \mathrm{mrad}$ and $>20$ detected photons/track were measured while $8.5 \mathrm{mrad}$ and $>20$ photons, respectively, are needed for a $3 \sigma \pi / K$ separation up to $3.5 \mathrm{GeV} / \mathrm{c}$. A decision between the bar and the cheaper plate option will be taken after more beam tests.

\section{References}

[1] PANDA Collaboration, Technical Progress Report, GSI, (2005); PANDA Collaboration, Physics Performance Report, (2009) arXiv:0903.3905v1.

[2] P. Coyle et al., Nucl. Instr. and Meth. A 343 (1994) 292.

[3] A. Lehmann et al., Nucl. Instr. and Meth. A 639 (2011) 144; F. Uhlig et al., Nucl. Instr. and Meth. A 695 (2012) 68.

[4] A. Lehmann et al., doi: 10.1016/j.nima.2014.04.005. 
[5] O. Merle et al., doi:10.1016/j.nima.2014.04.016.

[6] E. Etzelmüller et al., these proceedings.

[7] K. Föhl et al., Nucl. Instr. and Meth. A 732 (2013) 346.

[8] I. Fröhlich et al., IEEE Trans. Nucl. Sci. 5559 (2008); M Traxler et al., JINST 6 (2011), C12004;

C. Ugur et al., JINST 7 (2012), C02004.

[9] J. Schwiening et al., Nucl. Instr. and Meth. A 639 (2011) 315;

M. Hoek et al., doi:10.1016/j.nima.2014.04.006

[10] I. Adam et al., Nucl. Instr. and Meth. A 538 (2005) 281.

[11] M. Cardinali et al., doi:10.1016/j.nima.2014.04.071.

[12] R. Dzhygadlo et al., doi:10.1016/j.nima.2014.04.073.

[13] K. Inami et al., doi:10.1016/j.nima.2014.07.006.

[14] M. Starič, Nucl. Instr. and Meth. A 639 (2011) 252;

M. Starič, doi:10.1016/j.nima.2014.06.017.

[15] G. Kalicy et al., JINST 9 (2014), C05060, doi:10.1088/1748-0221/9/05/C05060.

[16] C. Schwarz et al., doi:10.1016/j.nima.2014.06.071. 